\title{
Estudio Ab-initio de propiedades estructurales, elásticas y electrónicas de nanohilos core/shell
}

\author{
Ab-initio study of structural, elastic and \\ electronic properties of core/shell \\ nanowires
}

Lucy Alejandra Valdez ${ }^{1}$, Ricardo Antonio Casali ${ }^{2}$

\author{
${ }^{1}$ Departamento de Física, Facultad de Ciencias Exactas y Naturales y Agrimensura, Universidad Nacional del Nordeste. \\ Av. Libertad 5450, CP: 3400, Corrientes, Argentina \\ e-mail: lucy.alejandra.valdez@gmail.com \\ ${ }^{2}$ Departamento de Física, Facultad de Ciencias Exactas y Naturales y Agrimensura, Universidad Nacional del Nordeste. \\ Av. Libertad 5450, CP: 3400, Corrientes, Argentina \\ e-mail: ra_casali@yahoo.com.ar
}

\section{RESUMEN}

El óxido de Zinc $(\mathrm{ZnO})$, por sus propiedades térmicas, electrónicas y piro-piezoeléctricas, es ampliamente usado en diversos dispositivos electrónicos. Algunas de estas propiedades mejoran notablemente en la nanoescala. Recientemente, se logró sintetizar nanohilos con estructuras core/shell de $\mathrm{ZnO} / \mathrm{X}$ ( $\mathrm{X}=\mathrm{ZnSe}, \mathrm{ZnS}$, $\mathrm{BeO})$ que mejoran la eficiencia de sensores piezoeléctricos, celdas fotovoltaicas y dispositivos optoelectrónicos. En este trabajo se presenta un estudio teórico de las propiedades estructurales, elásticas y electrónicas de nanoestructuras tipo core/shell de $\mathrm{ZnO} / \mathrm{X}(\mathrm{X}=\mathrm{ZnS}, \mathrm{BeO})$, cuando son sometidas a deformaciones uniaxiales en la dirección [0001]. Nanohilos hexagonales con diámetros entre 1.5 y $2.8 \mathrm{~nm}$ fueron estudiados ab-initio usando la teoría de la funcional de la densidad (DFT) en la aproximación de gradiente generalizado (GGA), implementada en el código SIESTA. Mediante la aplicación de tensiones uniaxiales, se calcularon el módulo de Young, la tensión de ruptura y las densidades de estados electrónicos totales (TDOS) y proyectados (PDOS). Se ha observado una degradación de las propiedades mecánicas en los nanohilos core/shell con respecto a los de $\mathrm{ZnO}$, ganándose a cambio la posibilidad de obtener un sistema con carácter más aislante o más semiconductor que el nanohilo de $\mathrm{ZnO}$, dependiendo si las capas externas están compuestas por $\mathrm{BeO}$ o $\mathrm{ZnS}$. Estos resultados son importantes para ajustar las propiedades optoelectrónicas del $\mathrm{ZnO}$ en la nanoescala.

Palabras claves: Nanohilos, core@ shell, ZnO, BeO, ZnS.

\section{ABSTRACT}

This Zinc oxide $(\mathrm{ZnO})$ is widely used in different electronic devices due to its thermal, electronic, and piropiezoelectric properties. Some of these properties are noticeable improvement at the nanoscale, specially when $\mathrm{ZnO}$ together with other material create heterostructures. Recently, core/shell nanowires have been synthesized in order to raise the performance in devices like piezo-electronic sensors, photovoltaic cells, and optoelectronic devices. In this work, we introduce a theoretical study of structural, elastic and electronic properties of core/shell nanostructures of $\mathrm{ZnO} / \mathrm{X}(\mathrm{X}=\mathrm{ZnS}, \mathrm{BeO})$ when they are subjected to uniaxial stresses in the [0001] direction. Hexagonal nanowires, whose diameters range from 1.5 to $2.8 \mathrm{~nm}$, were ab-initio studied using the density functional theory, in the generalized gradient approximation (GGA), implemented in the SIESTA code. By applying uniaxial stresses, we calculated the Young modulus, maximum tensile, and both total (TDOS) and projected electronic density of states (PDOS). We have observed a degradation in the mechanical properties of core/shell nanowires with respect to $\mathrm{ZnO}$ nanowires. Depending on the external shells (BeO or $\mathrm{ZnS}$ ), the core/shell nanowires became more insulator or more semiconductor. These results are important in nano electronic devices based on $\mathrm{ZnO}$, in order to tailor their optoelectronic properties.

Keywords: Nanowires, core@shell, ZnO, BeO, ZnS. 


\section{INTRODUCCIÓN}

En el campo tecnológico existen semiconductores destacables debido a su importancia en la industria y a su potencial utilidad como nano generadores de energía. Un ejemplo es el óxido de zinc ( $\mathrm{ZnO})$, el cual presenta estructura wurtzita (B4) a temperatura ambiente, y por sus propiedades térmicas, electrónicas y piropiezoeléctricas en dicha fase, es usado en diversos dispositivos electrónicos, como por ejemplo actuadores, sensores, etc. [1,2].

Numerosas investigaciones desarrolladas desde las últimas décadas evidencian que las propiedades de un material, como las mecánicas y electrónicas, cambian cuando este forma una nanoestructura. En particular, el $\mathrm{ZnO}$ mejora algunas de sus características cuando forma nanohilos [3-5] y en años recientes, se han sintetizado nanohilos tipo core/shell de $\mathrm{ZnO} / \mathrm{X}(\mathrm{X}=\mathrm{ZnSe}, \mathrm{ZnS}$, BeO) que aumentan la eficiencia de celdas fotovoltaicas y dispositivos optoelectrónicos [6-10], pueden utilizarse como biosensores de colesterol [11] y constituyen heteroestructuras prometedoras para dispositivos nanofotónicos y sensores con aplicaciones en la región infrarroja del espectro electromagnético [12]. Sin embargo, no se ha logrado un entendimiento completo de sus propiedades elásticas, mecánicas y electrónicas, lo cual es de fundamental importancia no sólo para comprender los fenómenos en la nanoescala sino también para proponer materiales alternativos beneficiosos para el uso humano.

Por este motivo, en el presente trabajo se expone un estudio ab-initio de las propiedades elásticas y electrónicas de los nanohilos de $\mathrm{ZnO}$ cuando son recubiertos por capas de $\mathrm{ZnS}$ y BeO. Nuestro objetivo principal es contribuir a una mejor comprensión de las propiedades elásticas y electrónicas de los nanohilos tipo core/shell, que son candidatos potenciales a constituir la próxima generación de nano dispositivos.

\section{MATERIALES Y MÉTODOS}

Se generaron nanohilos de $\mathrm{ZnO}$ de área transversal hexagonal, con diámetros (d) comprendidos entre 1,5 y 2,8 nm (Fig. 1) y con una altura de 1c, siendo c la longitud de la celda unitaria a lo largo de la dirección [0001]. La estructura cristalina corresponde a la fase wurtzita, siendo la constante de red optimizada a=0,32-, 0,38- y 0,28- nm para el material bulk de $\mathrm{ZnO}, \mathrm{ZnS}$ y $\mathrm{BeO}$, respectivamente.

Los sistemas core@shell aquí estudiados se componen de un core de 48 átomos rodeados por una y dos capas (shell) de material $\mathrm{X}(\mathrm{X}=\mathrm{ZnS}, \mathrm{BeO})$. Las respectivas celdas unitarias se presentan en la Fig.1 (A la izquierda se tiene un nanohilo con repetición de la celda unitaria en la dirección [0001]. A la derecha se observan las celdas unitarias de los sistemas core/shell, originales y optimizadas).

Los nanohilos fueron estudiados ab-initio con el código SIESTA [13] mediante la teoría de la funcional de la densidad (DFT) en la aproximación de gradiente generalizado (GGA).
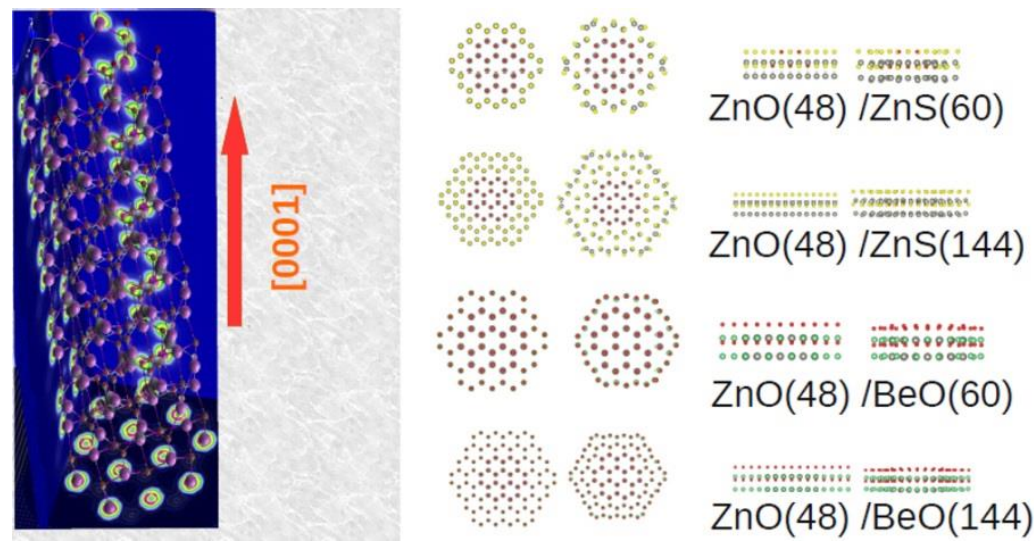

Figura 1: Celdas unitarias de los nanohilos. A izquierda se observa una repetición de la celda unidad a lo largo de la dirección [0001]. A derecha se presentan las celdas unidades (sin/con optimización) de los nanohilos core/shell vistos transversal y lateralmente a la dirección [0001], donde los átomos grises, rojos, amarillos y verdes son los de Zn, O, S y Be, respectivamente.

Mediante la aplicación de un conjunto de tensiones uniaxiales en la dirección [0001], se calcularon propiedades elásticas como el Módulo de Young $(\mathrm{Y})$ en el régimen lineal y el coeficiente de Poisson $(\sigma)$. 
Asimismo, se estimó la energía de la banda prohibida $\left(\mathrm{E}_{\mathrm{g}}\right)$ y se analizaron las densidades de estados electrónicos totales (TDOS) y proyectadas (PDOS) por capas atómicas.

\section{RESULTADOS Y DISCUSIONES}

En la Tabla 1 se resume las propiedades destacables de los nanohilos estudiados en el presente trabajo, indicándose número de átomos en la celda, diámetro (d), Módulo de Young $(\mathrm{Y})$, coeficiente de Poisson ( $\sigma$ ), tensión máxima $\left(\mathrm{T}_{\mathrm{max}}\right)$, deformación máxima $\left(\mathrm{e}_{\max }\right)$, y energía de gap $\left(\mathrm{E}_{\mathrm{g}}\right)$.

Con respecto al módulo de Young, nuestros cálculos están en el mismo orden de magnitud tanto experimental, reportado por CHEN et al. [5] de 208- y 220- GPa para nanohilos de $16 \mathrm{~nm}$ de diámetro, como teórica, reportado por HU et al. [14] de $213 \mathrm{GPa}$ para nanohilos de 1,7 nm de diámetro. KUNA et al. [15] obtuvieron $170 \mathrm{GPa}$ desde sus simulaciones de nanohilos de $100 \mathrm{~nm}$ de diámetro. En comparación con el material bulk de ZnO (140 GPa obtenido por KOBIAKOV [16], WANG et al. [3] en nanohilos de diámetros mayores a $200 \mathrm{~nm}$ y ROY et al. [17] en nanohilos de 78 a $310 \mathrm{~nm}$ de diámetro), el módulo de Young aumenta conforme disminuye el diámetro, sin embargo esta tendencia se ve alterada en los core/shell si el shell es $\mathrm{ZnS}$ o $\mathrm{BeO}$, produciéndose un descenso en el valor de $\mathrm{Y}$ que juntamente con la disminución de $\sigma$, indican que el sistema core/shell tendría un carácter más frágil que el correspondiente nanohilo de $\mathrm{ZnO}$.

Tabla 1: Propiedades básicas de los nanohilos estudiados, entre paréntesis se indica el número de átomos de cada compuesto.

\begin{tabular}{l|l|l|l|l|l|l}
\hline \multirow{2}{*}{ PROPIEDADES } & \multicolumn{3}{c}{ ZNO } & \multicolumn{5}{c}{ ZNO(48)@ } \\
\cline { 4 - 7 } & \multicolumn{2}{|c}{ ZNS(60) } & \multicolumn{1}{c}{ ZNS(144) } & \multicolumn{1}{c}{ BEO(60) } & BEO(144) \\
\hline NRO. DE ÁTOMOS & 108 & 192 & 108 & 192 & 108 & 192 \\
\hline $\mathrm{d}(\mathrm{nm})$ & 1,64 & 2,31 & 1,85 & 2,67 & 1,53 & 2,1 \\
\hline $\mathrm{Y}(\mathrm{GPa})$ & 217 & 201 & 135 & 106 & 155 & 219 \\
\hline$\sigma$ & 0,447 & 0,358 & 0,313 & 0,249 & 0,392 & 0,251 \\
\hline $\mathrm{T}_{\mathrm{MAX}}(\mathrm{GPa})$ & 34 & 31 & 10 & 12 & 26 & 20 \\
\hline $\mathrm{E}_{\mathrm{MAX}}$ & 0,171 & 0,146 & 0,219 & 0,338 & 0,350 & 0,385 \\
\hline $\mathrm{E}_{\mathrm{G}}(\mathrm{eV})$ & 1,3 & 1,2 & 0,64 & 0,51 & 2,42 & 2,6 \\
\hline
\end{tabular}

Por otro lado, pese a la degradación de las propiedades mecánicas, se puede obtener un sistema de carácter más aislante o más semiconductor, dependiendo del tamaño y composición de las capas de átomos que recubren al nanohilo de $\mathrm{ZnO}$, lo cual se refleja en los valores de la energía $\mathrm{E}_{\mathrm{g}}$ : cuando el material del shell es $\mathrm{ZnS}, \mathrm{E}_{\mathrm{g}}$ del sistema core/shell disminuye conforme se adhieren capas atómicas, y se tiene un efecto opuesto si el shell es BeO. Esta modulación de las propiedades elásticas y electrónicas, en conjunto con la ausencia de segregación de fase entre $\mathrm{ZnO}$ y $\mathrm{BeO}$ inclusive para altas concentraciones de Be reportado por KIM et al. [18], abren un camino para la mejora de las propiedades aislantes de nanodispositivos de ZnO. Por otra parte, los sistemas $\mathrm{ZnO} / \mathrm{ZnS}$ serían idóneos para dispositivos donde se precise un material de carácter semiconductor. En este sentido, SCHRIER et al. [6], GHRIB et al. [9], y CAO et al. [19] mostraron que el uso de nanohilos $\mathrm{ZnO} / \mathrm{ZnS}$ en celdas fotovoltaicas permitiría incrementar la eficiencia de los mismos con respecto a los paneles fotovoltaicos diseñados con $\mathrm{ZnO}$ bulk. Además, RAI et al. [20] demostraron experimentalmente la eficiencia de los nanohilos $\mathrm{ZnO} / \mathrm{ZnS}$ como dispositivos detectores de la radiación en el rango visible e infrarrojo del espectro electromagnético.

Dos propiedades importantes que varían con la tensión aplicada son $\sigma$ y $\mathrm{E}_{\mathrm{g}}$. En la Fig 2 se representa $\sigma$ en función de la deformación unitaria $(\alpha)$ a lo largo de la dirección [0001]. Claramente se observa una transición desde una estructura dúctil a frágil $(\sigma<0.25)$ conforme aumenta $\alpha$ para tres de los sistemas core/shell, excepto para el $\mathrm{ZnO}(48) / \mathrm{BeO}(144)$ cuyo comportamiento es diferente a los otros nanohilos. En el caso de $\mathrm{E}_{\mathrm{g}}$ se obtuvo una relación inversamente proporcional a $\alpha$. Recientemente, LUAN et al. [21] hallaron que en una monocapa de $\mathrm{ZnO}$ dopado con $\mathrm{Be}$, la $\mathrm{E}_{\mathrm{g}}$ se incrementa con el aumento en la concentración de Be conservando su característica de gap directo y se alcanza una mejor absorción óptica en la región ultravioleta respecto de la monocapa de $\mathrm{ZnO}$ sin dopaje. 
Con respecto a las propiedades electrónicas, se estudió la densidad de estados total (TDOS) (Fig.3) y las proyectadas (PDOS) por capas atómicas (Fig.4), enumerándolas desde el centro hacia el exterior del nanohilo y estableciéndose el nivel de Fermi en 0 eV. En el rango de energía comprendido entre -8 eV y el máximo de la banda de valencia, se observa la existencia de una banda de energía cuando el $\mathrm{ZnO}(48)$ se rodea con $\mathrm{BeO}$, situación que hemos observado en el nanohilo de $\mathrm{BeO}$; por el contrario, si el shell es $\mathrm{ZnS}$, dicha banda se desdobla en dos en las cercanías de los $-5 \mathrm{eV}$, lo cual fue congruente con nuestros nanohilos de $\mathrm{ZnO}$ y ZnS. Consecuentemente, la TDOS está representando el efecto combinado de dos materiales distinto.

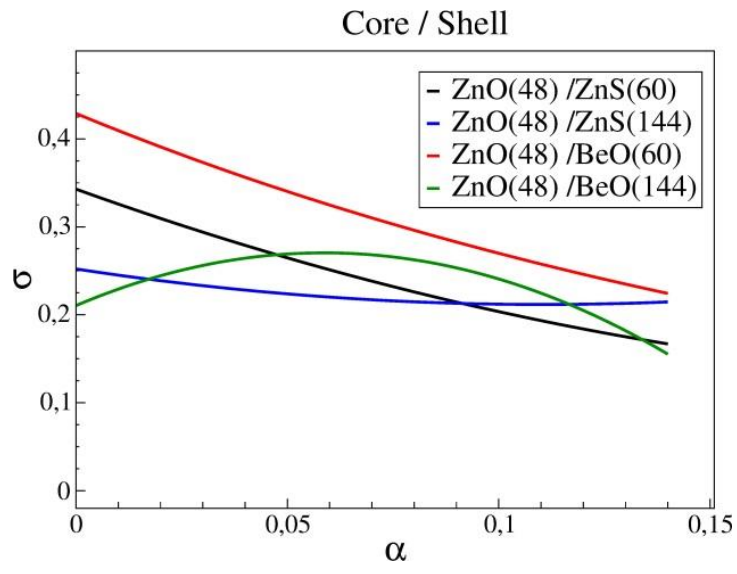

Figura 2: Coeficiente de Poisson vs deformación unitaria.

Para aclarar este efecto, la Fig. 4 nos permite ver que la mayor contribución a la TDOS en las zonas próximas al gap proviene de las capas externas y que las capas internas son las contribuciones propias de los nanohilos de $\mathrm{ZnO}$. Por otra parte, es importante tener presente la diferencia estructural entre los materiales que constituyen el core y el shell, puesto que se tiene asociado un problema interfacial. Este efecto es característico de las heteroestructuras a causa de la reordenación de las cargas en la zona limítrofe de la interfase. En nuestro caso particular, para $\mathrm{ZnO}(48) / \mathrm{BeO}$, los átomos de $\mathrm{Zn}$ presentes en la capa 1 y 2, y los átomos de O de la capa 3 y 4 son los responsables de que se tenga una sola banda entre los $-8 \mathrm{eV}$ y $0 \mathrm{eV}$. Por lo tanto, son los que intervendrán mayormente en los efectos de la interfase.

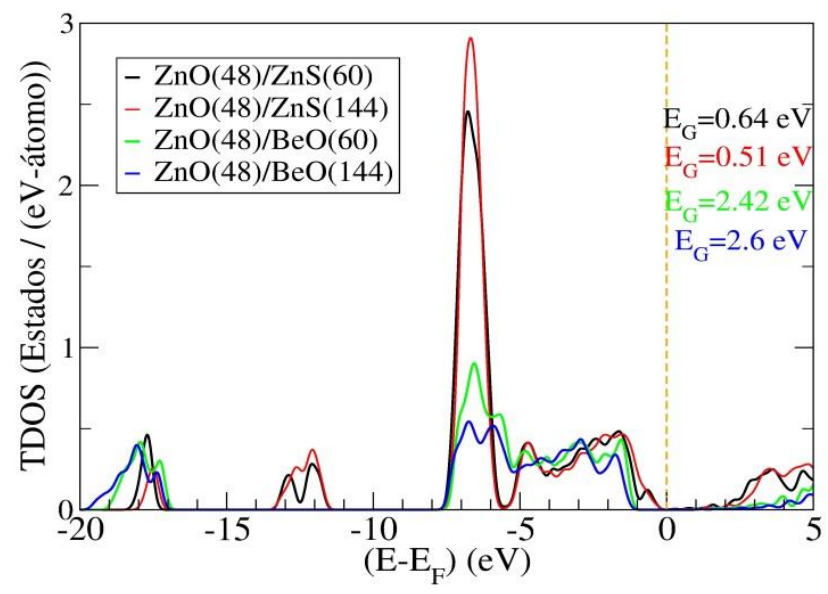

Figura 3: Densidad de estados totales (TDOS) de los nanohilos core/shell, normalizada por el número total de átomos.

Como era de esperarse, tanto en estos sistemas como en otras nanoestructuras, el aporte de los átomos de la superficie es importante en las zonas próximas a la energía del gap. 


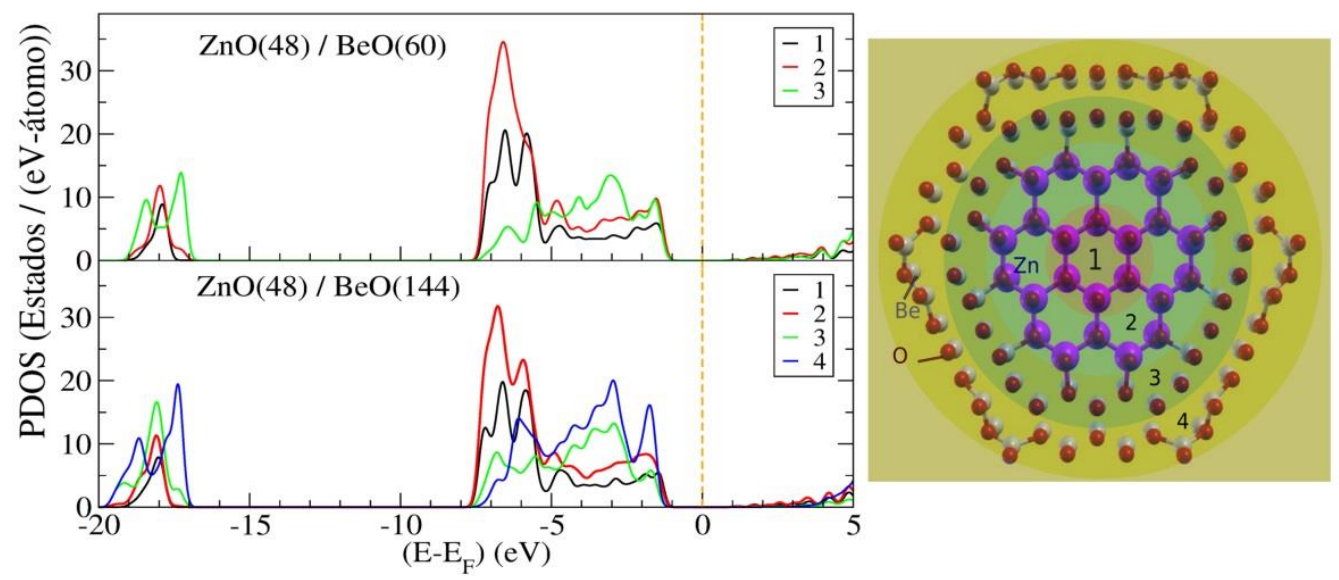

Figura 4: Densidad de estados proyectada (PDOS) de los nanohilos core/shell, normalizada por el número de átomos en cada capa. Composición de las capas: capa 1: $\mathrm{Zn}_{6} \mathrm{O}_{6}$; capa 2: $\mathrm{Zn}_{18} \mathrm{O}_{18}$; capa 3: $\mathrm{Be}_{30} \mathrm{O}_{30}$; capa 4: $\mathrm{Be}_{42} \mathrm{O}_{42}$.

\section{CONCLUSIONES}

El nanohilo de $\mathrm{ZnO}$ presenta disminuciones del módulo de Young y del coeficiente de Poisson, si adicionamos monocapas de $\mathrm{ZnS}$ o $\mathrm{BeO}$. Esto produce un comportamiento menos rígido y más frágil cuando el sistema se somete a tensiones uniaxiales en la dirección [0001]. Por otra parte, los nanohilos de $\mathrm{ZnO}$ mejoran su capacidad de conducción cuando son recubiertos por $\mathrm{ZnS}$ ( $\mathrm{E}_{\mathrm{g}}$ disminuye). Si el material de cobertura es $\mathrm{BeO}$ sucede lo opuesto, y el sistema presenta un carácter aislante ( $\mathrm{E}_{\mathrm{g}}$ aumenta con el espesor de $\left.\mathrm{BeO}\right)$. De esta manera, con el agregado de shell de los materiales descritos, es posible modular las propiedades optoelectrónicas de los nanohilos de $\mathrm{ZnO}$, en la nanoescala. Además, el sistema core/shell podría utilizarse para ajustar los procesos de transporte unidireccionales.

\section{AGRADECIMIENTOS}

L. A. Valdez desea agradecer al Consejo Nacional de Investigaciones Científicas (CONICET), por la beca doctoral otorgada. Los autores agradecen además las discusiones mantenidas con la Dra. María A. Caravaca (FI-UNNE).

\section{BIBLIOGRAFÍA}

[1] PRETE, P, Nanowires, 1 ed., Croatia, Intech, Published under CC BY-NC-SA 3.0 license. DOI: 10.5772/3457, 2010.

[2] ZHU, YONG, "Mechanics of Crystalline Nanowires: An experimental perspective", Applied Mechanics Review, v. 69, pp. 010802 (1-24), Jan. 2017.

[3] WANG, X., CHEN, K., ZHANG, Y., et al., "Growth conditions control the elastic and electrical properties of ZnO nanowires”, Nano Letters, v. 15, n. 12, pp. 7886-7892, Oct. 2015.

[4] WEI, B., ZHENG, K., JI, Y., et al., "Size-Dependent Bandgap Modulation of ZnO Nanowires by Tensile Strain”, Nano Letters, v. 12 , pp. 4595-4599, Ago. 2012.

[5] CHEN, C Q., SHI, Y., ZHANG, Y. S., et al., "Size dependence of Young's modulus in ZnO nanowires", Physical Review Letters, v. 96, n. 7, pp. 075505(1-4), Feb. 2006.

[6] SCHRIER, J., DEMCHENKO, D.O., WANG, L., et al., "Optical Properties of ZnO/ZnS and ZnO/ZnTe Heterostructures for Photovoltaic Applications”, Nano Letters, v. 7, n. 8, pp. 2377-2382, Jul 2007.

[7] ZHANG, Y., WU, Z., ZHENG, J., et al., “ZnO/ZnSe type II core-shell nanowire array solar cell”, Solar Energy Materials and Solar cells, v. 102, n. Supplement C, pp. 15-18, Jul. 2012.

[8] ZHOU, M., YI, Z., LI, K., et al., " Synthesis and characterization of aligned ZnO/BeO coreshell nanocable arrays on glass substrate”, Nanoscale Research Letters, v. 6, n. 1, pp. 506(1-6), Ago. 2011.

[9] GHRIB, T., AL-MESSIERE, M.A., AL-OTAIBI, A.L, "Synthesis and Characterization of $\mathrm{ZnO} / \mathrm{ZnS}$ Core/Shell Nanowires”, Journal of Nanomaterials, v. 2014, Article ID 989632, pp. 1-8, Feb. 2014.

[10] TARISH, S., AL-HADDAD, A., XU, R., et al., "The shift of optical absorption band edge of $\mathrm{ZnO} / \mathrm{ZnS}$ 
core/shell nanotube arrays beyond quantum effects", J. Mater. Chem. C, v. 4, n. 7, pp. 1369-1374, Jan. 2016. [11] GIRI, A.K., CHARAN, C., SAHA, A., et al., "An amperometric cholesterol biosensor with excellent sensitivity and limit of detection based on an enzyme-immobilized microtubular $\mathrm{ZnO} @ \mathrm{ZnS}$ heterostructure", J. Mat. Chem. A, v. 2, n. 40, pp. 16997-17004, Aug. 2014.

[12] D’AMICO, P., CALZOLARI, A., RUINI, A., et al., "New energy with ZnS: novel applications for a standard transparent compound", Scientific Reports, v. 7, n. 16805, pp. 1-8, Dic. 2017.

[13] SOLER, J.M., ARTACHO, E, GALE, J.D., et al., "The SIESTA method for ab initio order-N materials simulation"; Journal of Physics:Condensed Matter, v. 14, n. 11, pp. 2745-2779, Mar. 2002.

[14] HU, J., LIU, X.W., PAN, B.C., "A study of the size-dependent elastic properties of $\mathrm{ZnO}$ nanowires and nanotubes", Nanotechnology, v. 19, n. 28, pp. 1-6, Jun. 2008.

[15] KUNA, L., MANGERI, J., GAO, P., et al., "Stress-Induced Shift of Band Gap in ZnO Nanowires from Finite-Element Modeling", Phys. Rev. Applied, v. 8, n. 3, Sep. 2017.

[16] KOBIAKOV, I.B., "Elastic, piezoelectric and dielectric properties of $\mathrm{ZnO}$ and $\mathrm{CdS}$ single crystals in a wide range of temperatures", Solid State Communications, v. 35, n. 3, pp. 305-310, Jul. 1980.

[17] ROY, A., MEAD, J., WANG, S., et al., "Effects of surface defects on the mechanical properties of ZnO nanowire", Scientific Reports, v. 7, n. 9547, pp. 1-8, Ago. 2017.

[18] KIM, W. J., LEEM, J. H., HAN, M. S., et al., "Crystalline properties of wide band gap BeZnO films", Journal of Applied Physics, v. 99, n. 9, pp. 1-4, Mar. 2006.

[19] CAO, G., YANG, H., HONG, K., et al., "Synthesis of long $\mathrm{ZnO} / \mathrm{ZnS}$ core-shell nanowires and their optical properties", Materials Letters, v. 161, pp. 278-281, Dic. 2015.

[20] RAI, S.C., WANG, K., DING, Y., et al., "Piezo-phototronic Effect Enhanced UV/Visible Photodetector Based on Fully Wide Band Gap Type-II ZnO/ZnS Core/Shell Nanowire Array", ACS Nano, v. 9, n. 6, pp. 6419-6427, Jun. 2015.

[21] LUAN, Z., SUN, D., TAN, C., et al., "First-principles calculations of electronic structure and optical properties of Be-doped ZnO monolayer", Integrated Ferroelectrics, v. 179, n. 1, pp. 84-94, Aug. 2017. 